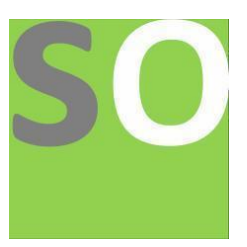

Article title: Image classification using concatenated of co-occurrence matrix features and local ternary patterns Authors: Faeze Kiani[1]

Affiliations: Department of electronic engineering, Online computer vision research group[1]

Orcid ids: 0000-0002-0654-2252[1]

Contact e-mail: f.kiani_ocvrg@yahoo.com

License information: This work has been published open access under Creative Commons Attribution License http://creativecommons.org/licenses/by/4.0/, which permits unrestricted use, distribution, and reproduction in any medium, provided the original work is properly cited. Conditions, terms of use and publishing policy can be found at https://www.scienceopen.com/.

Preprint statement: This article is a preprint and has not been peer-reviewed, under consideration and submitted to ScienceOpen Preprints for open peer review.

DOI: 10.14293/S2199-1006.1.SOR-.PPLMNCJ.v1

Preprint first posted online: 15 March 2021

Keywords: Image processing, Feature extraction, Texture Classification, Local Ternary Pattern, Co-occurrence matrix 


\title{
Image classification using concatenated of co- occurrence matrix features and local ternary patterns
}

\author{
Faeze Kiani ${ }^{1}$ \\ Department of electronic engineering, Online computer vision research group \\ F.Kiani_OCVRG@yahoo.com
}

\begin{abstract}
Texture, color, and shape are the three main components that the human visual brain uses to identify or identify environments and objects. Therefore, tissue classification has been considered by many scientific researchers in the last decade. The texture features can be used in many different vision and machine learning problems. As of now, various methods have been proposed for classifying tissues. In all methods, the accuracy of the classification is a major challenge that needs to be improved. This article presents a new method based on a combination of two efficient tissue descriptors, the co-occurrence matrix and local ternary patterns (LTP). First, the local binary pattern and LTP are performed to extract information from the local tissue. In the next step, a subset of statistical properties is extracted from the gray surface concurrency matrices. Finally, the interconnected features are used to teach classification. Performance is evaluated for accuracy on the Brodatz reference data set. The experimental results show that the proposed method offers a higher degree of classification compared to some advanced methods.
\end{abstract}

Keywords: Image processing, Feature extraction, Texture Classification, Local Ternary Pattern, Co-occurrence matrix

\section{Introduction}

Color, texture, shape and geometric properties are the main components used by the human visual system to identify environments and objects [1]. In about $65 \%$ of cases, texture plays the most important role in distinguishing objects and content over color and shape. Therefore, tissue classification has been considered by many scientific researchers in the last decade. Tissue characteristics can be used in many displacement vision problems and machine learning, such as medical image recognition [2-3], image retrieval [4], object recognition [5], skin recognition [6] and ..... In the last decade, texture classification and texture analysis have been an important topic in the literature on computer vision and image processing sciences. Because texture classification is closely related to sciences like machine learning, it works in areas like pattern recognition, object tracking, flaw detection, face tracking, and more. The main problem in classifying textures is related to two questions: 1 . Finding and introducing the best characteristics to describe the texture. 2. Select the best and most appropriate type of classification with the selected characteristics. We have seen a variety of debates on these two topics. Some of these methods work directly on images taken from textures, such as texture classification based on the random threshold vector technique [7] and texture classification based on primary pattern units [8]. Another group of methods first processes images and then looks for appropriate properties related to class labels, such as the classification of textures using locally advanced binary patterns, and the spatial distribution of dominant patterns [9] and a texture. New approach to inspection of industrial color products [10]. Because the methods presented so far are practical and appropriate in all cases, they are not guaranteed to work effectively in other programs. For this reason, the main objective of the researcher is to define new methods and characteristics. This article introduces a new method that acts as a general method. It can be used for a variety of applications, but it works well for rock texture classification and can accurately classify rock texture types using a single train pass. In this method, the image is first processed using LBP and GLCM algorithms. Then edge detection is done using edge filters on the image. We can create a suitable data set by extracting well-known statistical characteristics such as energy, entropy, and contrast. Finally, we can classify test data very precisely using classifiers. The reminder of this article is as follows: The section is related to the literature review. The LBP, LTP, and GLCM trends are described in this section. In section three, the proposed texture classification method is clearly described in detail. The experimental results are included in Section Four. Finally, the conclusions and future work are presented in Section 5.

\section{Literature review}

The main contribution of this paper is to present a combinational method for texture classification. In this respect, some efficient structural and statistical descriptors are used. In this section, the main computation process of used operators is described with details. 


\section{II.I. Basic local binary patterns (LBP)}

Local Binary pattern (LBP) is one of the most popular texture analysis operators that was introduced in [11]. It is a gray-scale invariant texture measure computed from the analysis of a $\mathrm{N} \times \mathrm{N}$ local neighborhood over a central pixel. LBP generates a binary code by thresholding a local center neighborhood by the gray value of its center. The neighbors are labeled using a binary code $\{0,1\}$ obtained by comparing their values to the central pixel value. If the neighbor's intensity is below the intensity of the central pixel, then it is labeled 0 , otherwise it is assigned the value 1:

$$
P_{i}^{\prime}=\left\{\begin{array}{rr}
0 & \text { if } I\left(x_{i}, y_{i}\right)<I\left(x_{0}, y_{0}\right) \\
1 & \text { Otherwise }
\end{array}\right.
$$

$P_{i}$ is the obtained binary code, $P_{i}$ is the original pixel value at position $i$ and $P_{0}$ is the central pixel intensity. The obtained value is then multiplied by weights given to the corresponding pixels. The weight is given by the value $2^{\mathrm{i}-1}$. Summing the obtained values gives the measure of the LBP:

$$
L B P=\sum_{i=1}^{N} P_{i}^{\prime} 2^{i-1}
$$

Where, $\mathrm{N}$ is the total number of neighbors. An example on how to compute LBP is shown in the figure 1 . The neighborhood size is $3 \times 3$ in the Fig 1 .

\begin{tabular}{|c|c|c|}
\hline 8 & 22 & 12 \\
\hline 93 & 50 & 55 \\
\hline 8 & 89 & 5 \\
\hline
\end{tabular}

\begin{tabular}{|l|l|l|}
\hline 0 & 0 & 0 \\
\hline 1 & $C$ & 1 \\
\hline 0 & 1 & 0 \\
\hline
\end{tabular}

\begin{tabular}{|c|c|c|}
\hline 32 & 64 & 128 \\
\hline 16 & $C$ & 1 \\
\hline 8 & 4 & 2 \\
\hline
\end{tabular}

$\operatorname{LBP}(C)=00010101=21$

Figure1. Computation LBP

The obtained compared matrix is multiplied by respected weight matrix. The weights kernel is shown in the Fig 1. The sum of the resulting values gives the LBP measure is 21 . The central pixel is replaced by the obtained value. By applying the LBP operator on the whole image, a new matrix is calculated.

\section{II.II. Local ternary patterns}

Basic version of the LBP is sensitive to impulse noise [12]. So, improved version LTP proposed for the first time in [13]. In LTP operator, for each pixel of an image a local ternary pattern is computed which is a 3-valued codes $(-1,0,1)$. The LTPs is defined as follows:

$\mathrm{LTP}_{\mathrm{P}, \mathrm{R}}=\sum_{\mathrm{i}=0}^{\mathrm{P}-1} \mathrm{~s}\left(\mathrm{~g}_{\mathrm{i}}-\mathrm{g}_{\mathrm{c}}\right) 3^{\mathrm{i}}$,

$$
s(x)\left\{\begin{array}{rll}
1 & \text { if } & x>=t \\
0 & \text { if } & |x|<t \\
-1 & \text { if } & x<=-t
\end{array}\right.
$$

Where, $t$ represents the user threshold for encoding. The output of the LTPP operator, $\mathrm{R}$ is a binary P-bit with different $3 \mathrm{P}$ values for each pixel in the image. This increases computational complexity, so for simplicity and to reduce computational complexity, the ternary code is divided into high LBP and low LBP. An example of the LTP encryption method and its division into upper and lower patterns is shown in Figure (2).

$$
\begin{gathered}
\mathrm{T}=8 \quad \begin{array}{|c|c|c|}
\hline 8 & 22 & 12 \\
\hline 93 & 50 & 55 \\
\hline 8 & 89 & 5 \\
\hline & \\
\hline-1 & -1 & -1 \\
\hline-1 & C & 0 \\
\hline-1 & +1 & -1 \\
\hline
\end{array}
\end{gathered}
$$

Ternary Code $=(-1)(-1)(-1)(-1)(-1) 1(-1) 0$

Upper binary code $=00000100=4$

Lowe binary code $=11111010=250$

Figure 2. A visual example of LTP encoding procedure 
In the end, the two upper and lower local binary patterns histograms are generated individually and connected together to produce a single histogram. LTP provide more discriminative features than LBP and some other version of LBP-based on reported results in recent papers [14-16].

\section{III. Gray Level Co-occurrence Matrix}

Gray Level Co-occurrence Matrix (GLCM) was proposed in [17] by Haralick and Shanmugam. It is very useful in texture analysis. It calculates the second order statistics related to image properties by considering the spatial relationship of pixels. GLCM depicts how often different combinations of gray levels co-occur in an image[1819].

GLCM is created by calculating how often a pixel of intensity $i$ is generated in a specific spatial relationship with a pixel of value $\mathrm{j}$. The spatial relationship can be determined in several ways, the default relationship is between a pixel and its immediate neighbor to its right. However, we can determine this relationship with different offsets and angles. The pixel at position $(i, j)$ in GLCM is the sum of the number of times the relationship ( $i, j$ ) occurs in the image. Figure 3 describes how to calculate GLCM. This displays an image and its corresponding sync matrix using the default spatial relationship pixel (offset $=1$ in the $\mathrm{x}$ direction). For this pair $(2,1)$ (pixel 2 is followed by pixel 1 to its right), it is found 2 times in the image, then the GLCM image has 2 as values in the position corresponding to the matrix $\mathrm{Ii}=1$ and $\mathrm{Ij}=2$. GLCM is a $256 \times 256$ matrix. Ii and Ij are the intensity values for an 8-bit image. GLCM can be calculated for eight directions around the desired pixel (Figure 3). The results of the sum in different directions lead to isotropic GLCM and help to achieve a constant rotation of GLCM (Figure 4).

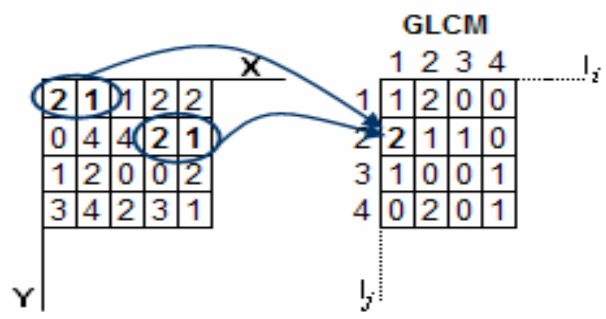

Figure3. A numric example of GLCM procedure

\begin{tabular}{|c|c|c|}
\hline $135^{\circ}$ & $90^{\circ}$ & $45^{\prime}$ \\
\hline $180^{\prime}$ & $\mathrm{x}$ & $0^{\prime}$ \\
\hline $225^{\circ}$ & $270^{\prime}$ & $315^{\prime}$ \\
\hline
\end{tabular}

Figure4. Eight popular directions used to generate for computing isotropic GLCM.

\section{Proposed texture classification method}

The proposed method is a multi-step method. First, the LBP operation is dedicated to the whole picture. The histogram of the filtered image with LBP output is considered a feature vector. Next, LTP is performed, which provides two vectors of different characteristics with $2 \mathrm{~N}$ dimensions. Where $\mathrm{N}$ represents the total number of neighbors according to the radius of the LBP neighborhood. For example, if a neighborhood of size 3.3 is considered, finally, the LBP histogram has 256 bins. Therefore, LBP provides a feature vector with 256 dimensions, and LTP extracts $2 \times 256$ features. In this step, the extracted feature vectors are combined. Next, a second-order statistic, called GLCM, is estimated for the original image. GLCM can be estimated in 8 directions and a matrix can be obtained in each direction. Finally, to achieve the differential properties, the 4 different popular statistical properties of each of the constructed GLCM matrices are calculated as follows:

$$
\begin{aligned}
& \text { Energy }=\sum_{i, j=0}^{N-1}\left(K_{i, j}\right)^{2} \\
& \text { Contrast }=\sum_{i, j=0}^{N-1} K_{i j}(i-j)^{2} \\
& \text { Homogeneity }=\sum_{i, j=0}^{N-1} K_{i j} / 1+(i-j)^{2} \\
& \text { Entropy }=\sum_{i, j=0}^{N-1}-\operatorname{Ln}\left(K_{i, j}\right) K_{i, j}
\end{aligned}
$$

$\mathrm{Ki}, \mathrm{j}$ is the value of the array at position $(\mathrm{i}, \mathrm{j})$ in the GLCM output array. $\mathrm{N}$ is the number of gray areas in the output image. Therefore, the 8 ويز 8 property 4 is calculated using GLCM. In this sense, each texture image can be described with 800 attributes. To evaluate the texture classification, it is sufficient to use the numerical 
classification. In the results section, it is shown that using these features, the classification of textures can be done with high precision.

\section{Experimental Results}

The main objective of this document is to provide an efficient method for the classification of tissues. In this regard, a multi-step approach based on a combination of LBP, LTP and GLCM characteristics is proposed. In this section, to evaluate the performance of the proposed method, the Broadatz data set is used as one of the most advanced data sets in tissue analysis [20]. To assess performance, several classifications are performed, including K Nearest Neighbor (KNN), Naive Bayes, and Random Forest. The results are shown in Table 1. As can be seen, KNN with $\mathrm{K}=3$ provides the highest precision between classifiers. In all experiments, $50 \%$ of the brodatz samples are used as train sets and $50 \%$ of the other images are used as test sets. Some examples of Broadatz data sets are shown in Figure 5.

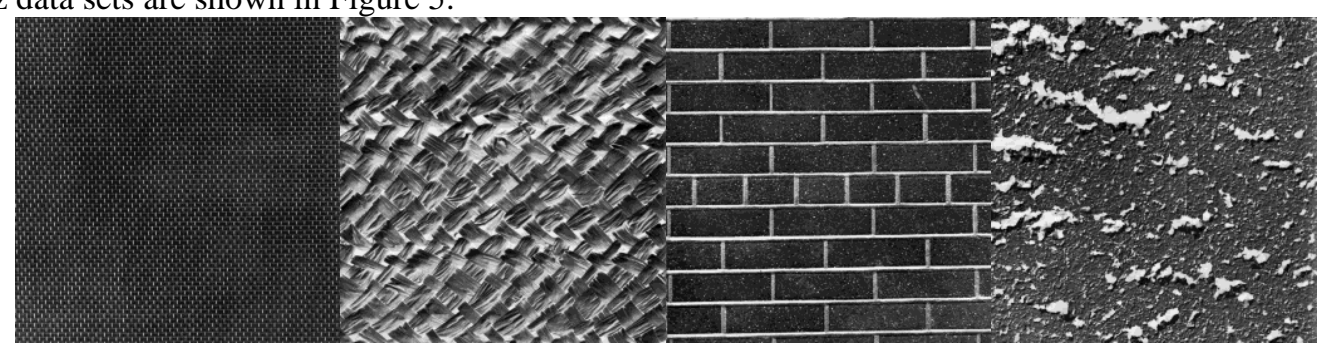

Figure 5. Some image samples of Brodatz dataset

\begin{tabular}{|c|c|c|c|c|c|}
\hline Classifier & $\begin{array}{c}\text { KNN } \\
\text { K=1 }\end{array}$ & $\begin{array}{c}\text { KNN } \\
\text { K=3 }\end{array}$ & $\begin{array}{c}\text { KNN } \\
\text { K=5 }\end{array}$ & $\begin{array}{c}\text { Naive } \\
\text { Bayes }\end{array}$ & $\begin{array}{c}\text { Random } \\
\text { forest }\end{array}$ \\
\hline $\begin{array}{c}\text { Approach } \\
\text { Proposed } \\
\text { approach }\end{array}$ & 89.12 & 92.76 & 92.24 & 79.43 & 88.34 \\
\hline
\end{tabular}

Since now, many different methods have been proposed for texture classification in gray-level images. So, the performance of our proposed method is compared with some of state-of-the-art methods in this scope. Compared results are shown in the table 2. As can be seen, in all cases, the proposed method provides higher performance in terms of classification accuracy.

Table2. Performance evaluation of proposed approach in comparison with state-of-the-art methods in terms of accuracy (\%)

\begin{tabular}{|c|c|c|c|c|c|c|}
\hline \multirow{2}{*}{$\begin{array}{c}\text { Method } \\
\text { Llassification } \\
\text { accuracy (\%) }\end{array}$} & 89.23 & 90.69 & 84.43 & 91.54 & 90.18 & LTP \\
\hline
\end{tabular}

\section{Conclusion}

The main contribution of this article was to propose a combination of textural descriptors and statistical specificity for tissue classification. In this sense, the multistage is presented based on a combination of local binary patterns, local ternary patterns, and statistical characteristics derived from GLCM. The results show that our proposed cocktail provides greater precision compared to many advanced methods in this field. Noise resistance is one of the main advantages of the proposed method due to the use of LTP. LBP and LTP are not sensitive to scaling and rotation. Eight addresses are used to perform GLCM, so the statistical properties extracted by GLCM are not sensitive to rotation. The feature vector proposed in this article is not unique, so it can be used in many other computer vision applications for the feature extraction step. As an idea for future work, the proposed method can be used in image retrieval systems in the learning phase to describe the content of the image.

\section{REFERENCES}

1. Jain, N., Sharma, S., and Sairam, R. M., "Content based image retrieval using combination of color, shape and texture features", International Journal of Advanced Computer Research, Vol. 3, No.1, pp. 70-77, 2013. 
2. Veerashetty, S., and Patil, N. B., "Novel LBP based texture descriptor for rotation, illumination and scale invariance for image texture analysis and classification using multi-kernel SVM, Multimedia Tools and Applications, Vol. 79, pp. 9935-9955, 2020.

3. Fekri-Ershad, S., "Pap smear classification using combination of global significant value, texture statistical features and time series features", Multimedia Tools and Applications, Vol. 78, No. 22, pp. 31121-31136, 2019.

4. Chavda, S. M., and Goyani, M. M., "Recent evaluation on Content Based Image Retrieval", International Journal of Computer Sciences and Engineering, Vol. 7, No. 4, pp. 325-329, 2019.

5. Yan, M., Li, Z., Yu, X., and Jin, C., "An End-to-End Deep Learning Network for 3D Object Detection From RGB-D Data Based on Hough Voting", IEEE Access, Vol. 8, pp. 138810-138822, 2020.

6. Fekri-Ershad, Sh., Saberi, M., and Tajeripour, F., "An innovative skin detection approach using color based image retrieval technique", International Journal of Multimedia \& Its Applications, Vol.4, No.3, pp. 57-65, 2012.

7. Reddy, B. V. R., Mani, M. R., Sujatha, B., and Kumar, V. V., "Texture Classification Based on Random Threshold Vector Technique", International Journal of Multimedia and Ubiquitous Engineering, Vol. 5, No. 1, 2010.

8. Cord, A., Bach, F., and Jeulin, D., "Texture classification by statistical learning from morphological image processing: application to metallic surfaces", Journal of Microscopy, Vol. 239, No. 2, pp. 159-166, 2010.

9. Liao, S., Law, M. W. K., and Chung, A. C. S., "Dominant Local Binary Patterns for Texture Classification", IEEE Transactions on Image Processing, Vol. 18, No. 5, pp. 1107-1118, 2009.

10. Fekri-Ershad, Sh., and Tajeripour, F., "Multi-resolution and noise-resistant surface defect detection approach using new version of local binary patterns", Applied Artificial Intelligence, Vol. 31, No. 5-6, pp. 395-410, 2017.

11. Ojala, T., Pietikainen, M., and Harwood, D., "A comparative study of texture measures with classification based on feature distributions", Pattern Recognition, Vol. 29, No. 1, pp. 51-59, 1996.

12. Tajeripour, F., Saberi, M., and Fekri-Ershad, Sh., "Developing a novel approach for content based image retrieval using modified local binary patterns and morphological transform", International Arab Journal of Information Technology, Vol. 12, No. 6, pp. 574-581, 2015.

13. Agarwal, M., Singhal, A., and Lall, B., "Multi-channel local ternary pattern for content-based image retrieval", Pattern Analysis and Applications, Vol. 22, pp. 1585-1596, 2019.

14. Sukhia, K. N., Riaz, M. M., Ghafoor, A., and Ali, S.S., "Content-based remote sensing image retrieval using multi-scale local ternary pattern", Digital Signal Processing, Vol. 104, pp. 102765, 2020.

15. Rassem, T. H., Makbol, N. M., and Yee, S. Y., "Face Recognition Using Completed Local Ternary Pattern (CLTP) Texture Descriptor", International Journal of Electrical and Computer Engineering, Vol. 7, No. 3, pp. 1594-1601, 2017.

16. Fekri-Ershad, S., "Bark texture classification using improved local ternary patterns and multilayer neural network", Expert Systems With Applications, Vol. 158, pp. 113509, 2020.

17. Haralick, R. M., Shanmugam, K., and Dinstein, I., "Textural Features for Image Classification", IEEE Transaction on Systems, Man and Cybernetics, Vol. 6, pp.610-621, 1973.

18. Zhang, X., Cui, J., Wnag, W., and Lin, C., "A study for texture feature extraction of high-resolution satellite images based on a direction measure and gray level co-occurrence matrix fusion algorithm", Sensors, Vol. 17, No. 7, pp. 1474, 2017.

19. Hor, N., and Fekri-Ershad, Sh., "Image retrieval approach based on local texture information derived from predefined patterns and spatial domain information", International Journal of Computer Science Engineering, Vol. 8, No. 6, pp. 246-254, 2019.

20. Cusano, C., Naopoletano, P., and Schettini, R., "Combining local binary patterns and local color contrast for texture classification under varying illumination", Journal Optical Society of America, Vol. 31, No. 7, pp. 14531461. 2014.

21. Pietikäinen, M., Ojala, T., and $\mathrm{Xu}, \mathrm{Z}$., "Rotation-Invariant Texture Classification Using Feature Distributions", Pattern Recognition, Vol. 33, pp. 43-52, 2000. 
22. Zulpe, N. and Pawar, V., "GLCM textural features for brain tumor classification", International Journal of Computer Science Issues (IJCSI), Vol. 9, No. 3, pp.354-359, 2012.

23. Ershad, S. F., "Texture classification approach based on combination of edge \& co-occurrence and local binary pattern", arXiv preprint arXiv:1203.4855. 2012.

24. Banerji, S., Sinha, A., and Liu, C., "New image descriptors based on color, texture, shape, and wavelets for object and scene image classification", Neurocomputing, Vol. 117, pp. 173-185, 2013.

25. Goncalves, H., Corte-Real, L., Goncalves, J. A., "Automatic image registration through image segmentation and SIFT", IEEE Transactions on Geoscience and Remote Sensing, Vol. 49, No. 7, pp. 2589-600, 2011.

27. Zhu, L., Shen, J., Xie, L., and Cheng, Z., "Unsupervised visual hashing with semantic assistant for contentbased image retrieval", IEEE Transaction on Knowledge and Data Engineering, Vol. 29, No. 2, pp. 472-486, 2017.

26. Bianconi, F., and Fernandez, A., "Evaluation of the effects of gabor filter paprameters on texture classification", Pattern Recognition, Vol. 40, No. 12, pp. 3325-33335, 2007.

28. Tajeripour F., Rezaei, M., Saberi, M., Fekri-Ershad, Sh., "Texture classification approach based on combination of random threshold vector technique and co-occurrence matrixes", In Proc. of International Conference on Computer Science and Network Technology, Harbin, China, pp. 2303-2306, 2011.

29. Fekri-Ershad, Shervan, and Farshad Tajeripour. "Impulse-Noise resistant color-texture classification approach using hybrid color local binary patterns and kullback-leibler divergence." The Computer Journal, Vol. 60, No. 11, pp. 1633-1648, 2017.

30. Guang, L., Lei, Z., Ying, H., Zuo-Yong, L., and Jing-Yu, Y., "Image Retrieval Based on Multi Texton Histogram", Pattern Recognition, Vol. 43, No. 7, pp. 2380-2389, 2010. 